\title{
Calculation Model for Travel Speed and Psychological State in Escape Routes considering Luminous Condition, Smoke Density and Evacuee's Visual Acuity
}

\author{
YUKI AKIZUKI ${ }^{1}$, TAKEYOSHI TANAKA ${ }^{2}$ and KOHEI YAMAO ${ }^{2}$ \\ 1: Faculty of Human Development, University of Toyama \\ 3190 Gofuku, Toyama, Toyama 930-8555, JAPAN \\ 2: Disaster Prevention Research Institute, Kyoto University \\ Gokasho, Uji, Kyoto 611-0011, JAPAN
}

\begin{abstract}
We conducted travel speed measurements to clarify the effect of visibility on evacuee's performance. As visual ability is dependent on the individual, members of the two age groups (30 youths and 30 seniors) were subjected to a visual acuity test prior to the travel experiment. In our research, we define subject's visual acuity as recognizable threshold of form perception using Landolt Ring. Visual acuity is a person's ability to see distinctly the details of an object. We set eight levels of floor illuminance, complete or incomplete adaptation conditions and luminous conditions with or without smoke. Since the travel speed of the younger group was faster than that of the older group, regardless of illuminance level or smoke density, the difference in travel speed can be predicted by visual acuity. In this report, we constructed a calculation model to predict travel speed as functions of the luminous environment (incorporating illuminance level, adaptation condition, and smoke density) and evacuee's visual acuity. This model helps us predict performance of evacuees under fire, smoke or blackout conditions.
\end{abstract}

KEYWORDS: human behavior, egress, travel speed, visibility

NOMENCLATURE LISTING

\begin{tabular}{llll}
$C s$ & smoke density $(1 / \mathrm{m})$ & \multicolumn{2}{l}{ subscripts } \\
$d$ & distance between phototube sensors $(\mathrm{m})$ & $a$ & adapting space \\
$E$ & illuminance $(\mathrm{lx})$ & age & aged group \\
$k$ & constant number of psychological state & $i$ & incomplete adaptation without smoke \\
$L$ & luminance $\left(\mathrm{cd} / \mathrm{m}^{2}\right)$ & $o$ & complete adaptation without smoke \\
$P$ & negative psychological response ratio & $s$ & complete adaptation with smoke \\
$R E_{\text {adaptation }}$ & Ratio of adaptation illuminance change & $t$ & travel space \\
$R V$ & ratio of travel speed & young & young group \\
$V$ & travel speed $(\mathrm{m} / \mathrm{s})$ & & \\
$V A$ & visual acuity & Greek & \\
$V_{o}$ & initial voltage of phototube $(\mathrm{mV})$ & $a$ & constant number of visual ability \\
$V$ & voltage of phototube $(\mathrm{mV})$ & $\rho$ & reflectance
\end{tabular}

\section{INTRODUCTION}

When a serious fire breaks out, causing extensive smoke spread or power failure, deterioration of the visibility of escape routes is expected. This deterioration of visibility may significantly influence the evacuation behavior of evacuees, and potentially result in serious human hazards.

On February 28, 2003, a major disaster occurred in Taegue, Korea, where an incident in a subway caused more than 200 deaths. More than half of the casualties died within the subway cars, and the rest in the process of evacuation on the subway premises. All of the casualties were killed because they could not find proper evacuation routes quickly enough to get out alive. This alarming accident demonstrates clearly that designing for emergency evacuation is crucial in order to avoid major loss of life.

Realizing that contemporary urban structures are complex and multistory, evacuation design is extensively researched. But there has been no work on the relation between an evacuee's behavior and his visual ability. Lowered visibility on escape routes in emergency situations in urban areas can induce human damage 
resulting from difficulty in prompt evacuation. Therefore, it is necessary to establish a method for calculating or predicting evacuation behavior based on evacuee's visual ability, i.e. visual acuity. In our research, we define subject's visual acuity as recognizable threshold of form perception using Landolt Ring. Visual acuity is a person's ability to see distinctly the details of an object.

We have done a series of studies [1] on visibility evaluation based on different visual abilities. Further on, this report studies the relationship between visibility of the environment and travel speed in a model corridor simulating evacuation routes.

\section{PREVIOUS STUDIES ON EVACUATION PERFORMANCE}

There are some important previous studies on evacuation performance. Simmons [2] studied the relationship between illuminance and travel speed of evacuees. The result was referenced by CIE standards of Emergency lighting [3], which set the standard value $1.0(\mathrm{~lx})$ of floor illuminance for $1.0(\mathrm{~m} / \mathrm{s})$ of travel speed. Jaschinski [4] compared the travel speed of young and elderly subjects. In his results, the elderly group walked slower than the young group. Webber [5] converted these results from travel time to travel speed versus floor illuminance (Fig.1). However, direct application of the findings to concrete design of evacuation routes may be difficult, since several points were left incompletely determined by their studies, that is,

(1) From their correlation in Fig.1, it is not clear at which illuminance value travel speed reaches the maximum.

(2) Effect of light adaptation on the travel speed under circumstances with sudden change of illuminance in the course of evacuation was not investigated.

(3) The relationship between travel speed and visual ability in smoke was not investigated.

Jin [6] conducted evaluation experiments on travel speed under smoke conditions, showing that travel speed decreased as smoke density increased, and that the ratio of the decrease was affected by the smoke's stimulus property.

However, since the effects of visual ability were left unexplored by his studies too, direct application of the findings to concrete design of evacuation routes may not be possible.

In order to resolve these problems and to establish a

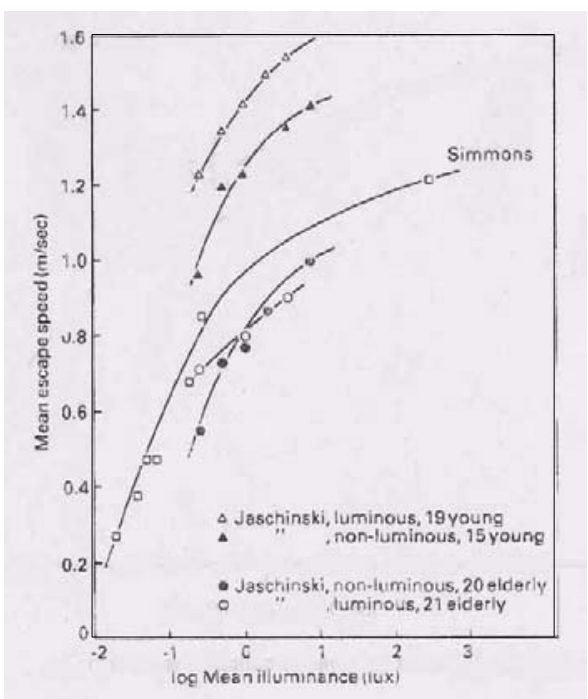

Fig.1. Previous study on travel speed [5]. method for calculating evacuation behavior based on an evacuee's ability to see in smoke, we conducted experiments to study the relationship among visual conditions, human visual ability and evacuation performance.

\section{EXPERIMENT}

\section{Apparatus}

Figure 2 shows the cross-section view of the experimental space, $1.77(\mathrm{~m})$ height and $1.77(\mathrm{~m})$ wide and $27.78(\mathrm{~m})$ length. Since the ceiling height of the travel space was limited, subjects shorter than $1.77(\mathrm{~m})$ were recruited as best as possible. Six subjects (out of 60 ) were found to be taller than $1.77(\mathrm{~m}$ ), so they had to bend down slightly when walking, but no particular difference in travel speed or questionnaire results was perceived between them and the others. The space was divided into three sections; adapting space (Length $5.58 \mathrm{~m})$, travel space $(18.48 \mathrm{~m})$ and questionnaire space $(3.72 \mathrm{~m})$. Figure 2 shows the cross-section view of the experimental space. Daylight was shut off by walls and a shading curtain and the reflectance ratio of all the wall surfaces was 0.43 .

Fluorescent lights of 20 (W) were installed at the corner of the ceiling and sidewall at $3.6(\mathrm{~m})$ intervals, securing even, distributed illuminance around the center of the floor. The lights of the adapting space and 
travel space were connected to respective dimmers, so we could control the floor illuminance level $\left(E_{t}\right)$ of each space separately from 0.03 (lx) to 300 (lx).

Infrared sensors were located on the sidewall at $3.0(\mathrm{~m})$ intervals to measure when a subject crossed the sensors. As the experimental space was a simple, straight passage, change of illuminance did not make a noticeable difference in travel speed in the preliminary experiment. So, to impose a visual task on subjects, cubic blocks (dimension $0.1(\mathrm{~m})$ or $0.05(\mathrm{~m})$, reflectance ratio 0.36 , luminance contrast with the floor 0.16 ) were randomly put on the floor. With this arrangement, the travel speeds became more dependent on the illuminance level due to the visual task that the subjects had to carefully watch and avoid kicking the blocks. Our experiment is not close enough to the fire emergency situations, so our results should be applied with careful consideration. However, the relationship between lighting environment and travel speed, taking into account visual acuity, was made more evident by our study.
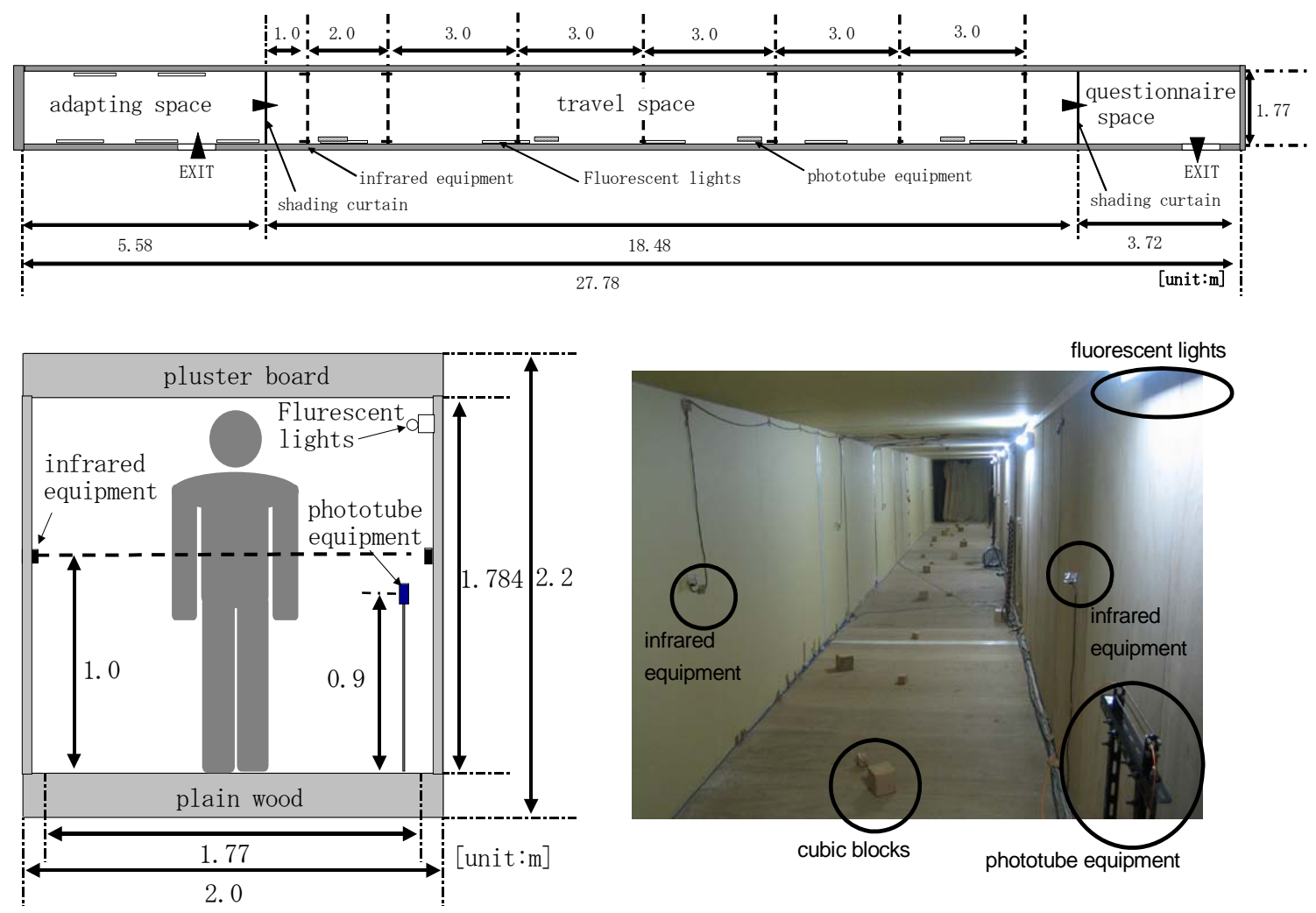

Fig. 2. Experimental apparatus.

\section{Experimental Procedure}

A subject was first exposed to the illuminance of the adapting space for 2.5 minutes, and then entered the travel space. He walked along the center line of the travel space, and negotiated the cubic blocks scattered about on the floor until he reached the questionnaire space. After entering the questionnaire space, he was asked to answer questions on "visibility of travel space", "ease to walking", and "anxiety while walking in the travel space" shown in Table 1. The questionnaire was designed to evaluate subjective and psychological states for investigating the relationship with travel speed.

Table 1. Categories of a psychological questionnaire

\begin{tabular}{|l|l|}
\hline \multicolumn{2}{|c|}{ "visibility of travel space" } \\
\hline \hline 0 & No problem \\
\hline 1 & a little difficult to see \\
\hline 2 & difficult to see \\
\hline 3 & very difficult to see \\
\hline 4 & cannot see \\
\hline
\end{tabular}

\begin{tabular}{|c|l|}
\hline \multicolumn{2}{|c|}{ "ease to walk or not" } \\
\hline \hline 0 & No problem \\
\hline 1 & a little difficult to walk \\
\hline 2 & difficult to walk \\
\hline 3 & very difficult to walk \\
\hline 4 & cannot walk \\
\hline
\end{tabular}

\begin{tabular}{|l|l|}
\hline \multicolumn{2}{|c|}{ "anxiety while walking in the travel space" } \\
\hline \hline 0 & No problem \\
\hline 1 & a little uneasy \\
\hline 2 & uneasy \\
\hline 3 & significantly uneasy \\
\hline
\end{tabular}




\section{Experimental Conditions}

Table 2 shows the luminous conditions in this series of experiments. We experimented on two types of adaptation: complete adaptation and incomplete adaptation. We set eight levels of floor illuminance in the travel space $\left(E_{t}\right): 0.03,0.1,0.3,1,3,10,30$, and $100(\mathrm{~lx})$. We treated the illuminance difference between the adapting space $\left(\mathrm{E}_{\mathrm{a}}\right)$ and the travel space as the ratio of adapting illuminance change $\left(\mathrm{RE}_{\text {adaptation }}\right)$ shown in Eq.1.

$$
R E_{\text {adaptation }}=\frac{E_{a}}{E_{t}}
$$

where the illuminance on the floor level of the adapting space is $E_{a}(l x)$, and the illuminance on the floor level of the travel space is $E_{t}(l x)$. We set the value of $R E_{\text {adaptation }}$ from 1 to 10000 . When the value of $\mathrm{RE}_{\text {adaptation }}$ is 1 , it means that the adaptation condition is complete and the illuminance of the travel space $\left(E_{t}\right)$ and the adapting space $\left(E_{a}\right)$ are at the same level.

Because of the high likelihood that evacuees in fire may have to travel in smoke, it is necessary to predict how smoke influences evacuees' performance. We used a smoke generator that could optionally adjust the discharge rate of white smoke in the experiment. The smoke was diffused evenly enough in the travel space using a fan. Smoke density in the travel space was measured by using four phototubes. We calculated smoke density scores under any illuminance condition by averaging all the data of each phototube every five seconds (Eq.2)

$$
C s=\frac{1}{d} \times \log _{e} \frac{V}{V_{o}}
$$

where the initial voltage of the phototube without smoke is $V_{o}(\mathrm{mV})$, the voltage of the phototube with smoke is $V(\mathrm{mV})$, and the distance between sensors in a phototube is $d(\mathrm{~m})$.

Smoke's influence on luminous conditions was compensated by the dimmer to secure the similar illuminance level of $0.03 \sim 100$ (lx). Control of the smoke density was extremely difficult, therefore the smoke density varied from 0.2 to $1.8(1 / \mathrm{m})$ depending on the experiment date, even if the floor illuminance in the travel space was the same. We used the dimmer to calibrate light so that the floor illuminance matched the values indicated in Table 2. The average density of over the entire experiment was $0.68(\mathrm{l} / \mathrm{m})$.

Table 2. Luminous conditions in this experiment.

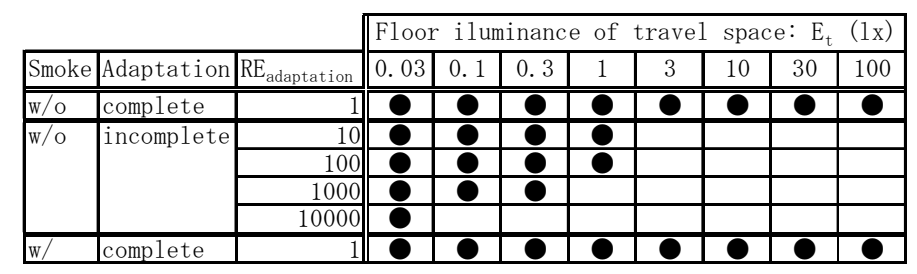

\section{Subjects}

\section{Age, Height and gender}

As visual ability is an independent variable, the two age groups (young and aged) were subjected to a visual acuity test prior to the travel experiment. Subjects consisted of 30 youths and 30 seniors. The young subjects were university students and research staff, and the aged subjects were registered at the Japan Senior Citizens Human Resource Center, a nationwide network providing seniors with temporary job opportunities. No subject had a walking impediment. Some subjects used visual correction (21 young and 14 aged used glasses or contact lens). These subjects need glasses or contact lens in daily life wore them in both the visual acuity test and the travel experiment. Subjects' age, height, and gender are shown in Table 3 and Fig. 3. 
Table 3. Subject.

\begin{tabular}{|c|c|c|c|c|}
\cline { 2 - 5 } \multicolumn{1}{c|}{} & \multicolumn{2}{c|}{ Young } & \multicolumn{2}{c|}{ Aged } \\
\cline { 2 - 5 } \multicolumn{1}{c|}{} & male & female & male & female \\
\hline number & 20 & 10 & 23 & 7 \\
\hline age & 24.2 & 26.4 & 71.0 & 67.7 \\
[years] & \pm 4.4 & \pm 5.7 & \pm 3.0 & \pm 2.7 \\
\hline height & 176.1 & 165.7 & 165.0 & 152.8 \\
[cm] & \pm 5.3 & \pm 4.7 & \pm 4.4 & \pm 4.0 \\
\hline
\end{tabular}

average \pm standard deviation

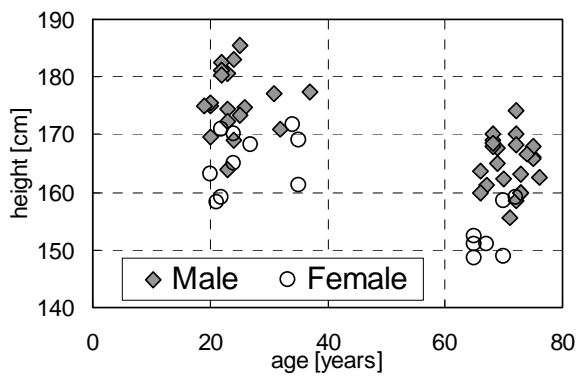

Fig. 3. Relationship between height and age of subjects.

\section{Visual Acuity}

Generally speaking, as shown in Fig. 4, three factors are closely related to visibility: environmental conditions, object's conditions and human visual ability. And we can predict the visibility of a visual environment using four elements: visual angle (minutes), background luminance $\left(\mathrm{cd} / \mathrm{m}^{2}\right)$, contrast (-) and visual acuity of an observer. In this research, we dealt with floor luminance of the walking space as the visual stimulus of the space under various lighting/smoke conditions, and tried to elucidate the age-related difference of travel speed in previous studies [4][5] using subjects' visual acuities.

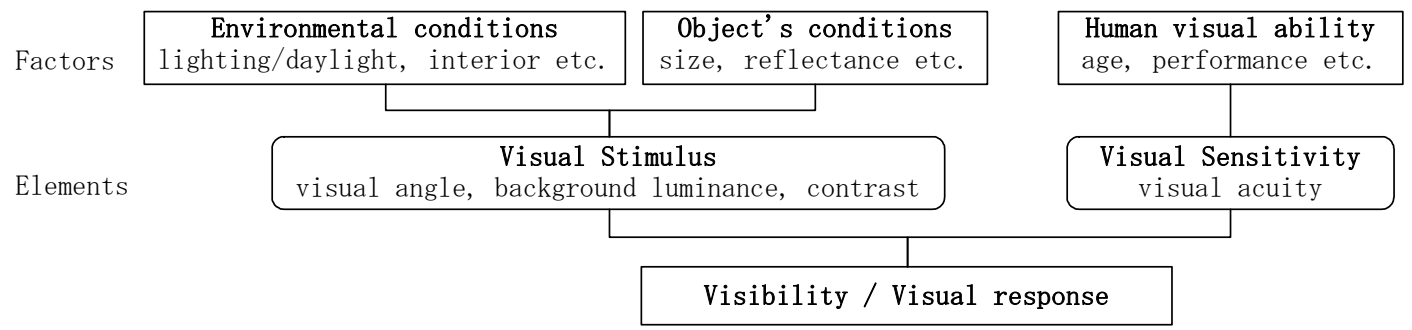

Fig.4. Model of evaluation system for visibility.

We measured the visual acuity of the subjects using a test chart of Landolt Ring in advance of the experiments. Subjects adapted to each illuminance level (from 0.1 to $1000 \mathrm{~lx}$ ) on the surface of the test chart for 2.5 minutes in a room where the visual acuity test was conducted. The wall reflectance of the measurement room was 0.93 , and luminous distribution over the test chart was almost even. The distance of the test chart from a subject was $1.5(\mathrm{~m})$. The background reflectance of the test chart was 0.70 , and the contrast of test chart between background and Landolt Ring was 0.94. Each Subject answered the gap direction of Landolt Ring, and his visual acuity under each illuminance level was determined by $80 \%$ of the correct answer ratio of 8 gap directions tested. No matter what the percentage of the correct answer ratio was, relations with the illuminance level were almost the same. Therefore, we show the results at $80 \%$ of the correct answer ratio, as this level is safe enough for application to design.

In designing the experimental luminous conditions, it was easier to use illuminance as the luminous value, and lighting design in buildings is based on floor illuminance. But in predicting visual acuity, it is more useful to use luminance than illuminance. We controlled the luminous level on the surface of the test chart by an illuminance meter, so we calculated the background luminance of the test chart from the surface illuminance and the reflectance ratio using Eq.3. The relationship between illuminance and luminance in this experiment is shown in Table 4.

$L=\frac{E \rho}{\pi}$

where luminance is $\mathrm{L}\left[\mathrm{cd} / \mathrm{m}^{2}\right]$, illuminance is E[lx], and reflectance ratio is $\rho[-]$. 
Table 4. Correspondence table between illuminance and luminance in this experiment.

\begin{tabular}{|c||c|c|c|c|c|c|c|c|c|c|}
\hline illuminance $[1 \mathrm{x}]$ & 0.03 & 0.1 & 0.3 & 1 & 3 & 10 & 30 & 100 & 300 & 1000 \\
\hline \hline $\begin{array}{c}\text { floor luminance }\left[\mathrm{cd} / \mathrm{m}^{2}\right] \\
\text { (reflectance 0.43) }\end{array}$ & 0.004 & 0.014 & 0.041 & 0.137 & 0.411 & 1.37 & 4.11 & 13.7 & - & - \\
\hline $\begin{array}{c}\text { test chart luminance }\left[\mathrm{cd} / \mathrm{m}^{2}\right] \\
\text { (reflectance 0.70) }\end{array}$ & - & 0.022 & 0.067 & 0.223 & 0.668 & 2.23 & 6.68 & 22.3 & 66.8 & 222.8 \\
\hline
\end{tabular}

Figure 5 shows the relationship between visual acuity and background luminance of the test chart for each age group. Visual acuity was enhanced with higher luminance. In Fig.5, there are large variations among individual results, but positive correlations were confirmed between visual acuity and background luminance. Equation 4 shows the relationship between luminance and visual acuity in the two age groups.

$$
V A=\alpha \times\left(\log _{10} L+1.85\right) \quad \because \alpha_{\text {aged }}=0.17, \alpha_{\text {young }}=0.34
$$

where visual acuity is $\mathrm{VA}[-]$, the background luminance of the test chart is $\mathrm{L}\left[\mathrm{cd} / \mathrm{m}^{2}\right]$, and age-related constant number is $\alpha$ [-]. Equation 4 shows that the visual acuity of the aged group consistently was about one half of that of the young group under any luminance levels. Therefore, representing visibility under light conditions meant considering age differences by visual acuity. By converting the floor illuminance of the travel space into luminance by using Table 4, it predicted levels of visual acuity under any experimental condition.
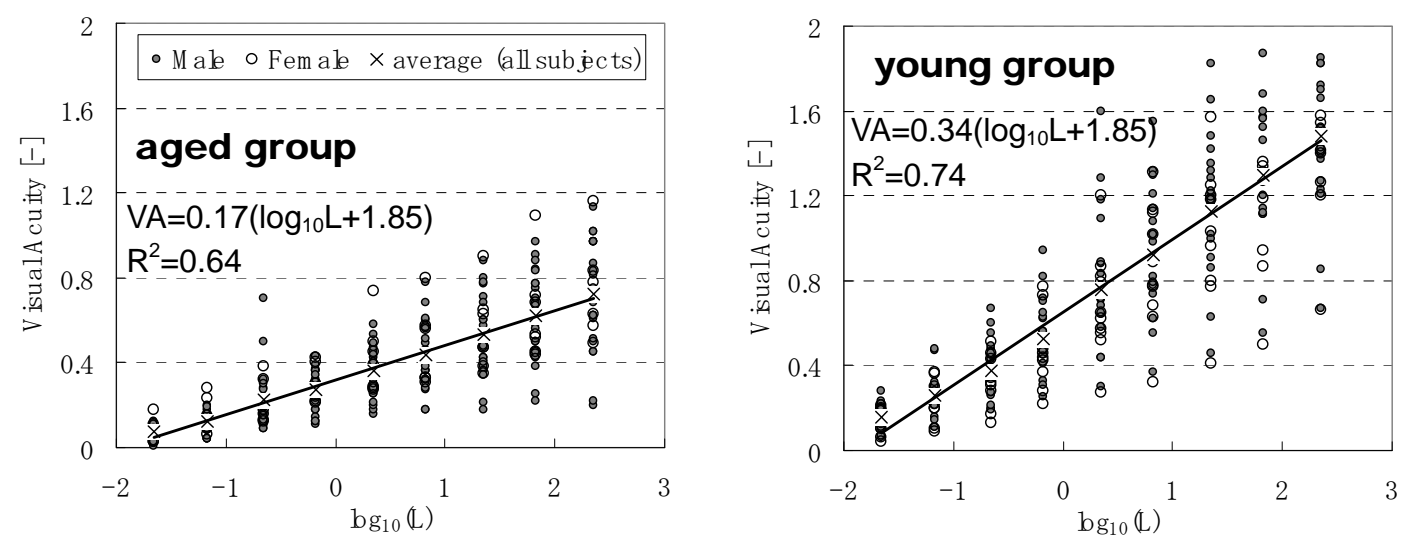

Fig.5. Relationship between background luminance and visual acuity.

\section{RELATIONSHIPS AMONG ILLUMINANCE, TRAVEL SPEED AND VISUAL ACUITY UNDER COMPLETE ADAPTATION}

The relationship between travel distance and speed in the travel space is shown in Fig. 6. No remarkable change of speed with travel distance is seen here, but the speed tended to increase with the level of the floor illuminance.

Fig.7 shows the relationship between travel speed and floor illuminance for the two age groups. At 1.0 (lx) of floor illuminance and lower, the average speed of young group was higher than aged group, which supports the findings of the previous study [2]. If the floor illuminance was higher than 3.0 [lx], the travel speed was similar for both age groups.

The relationship between visual acuity and travel speed

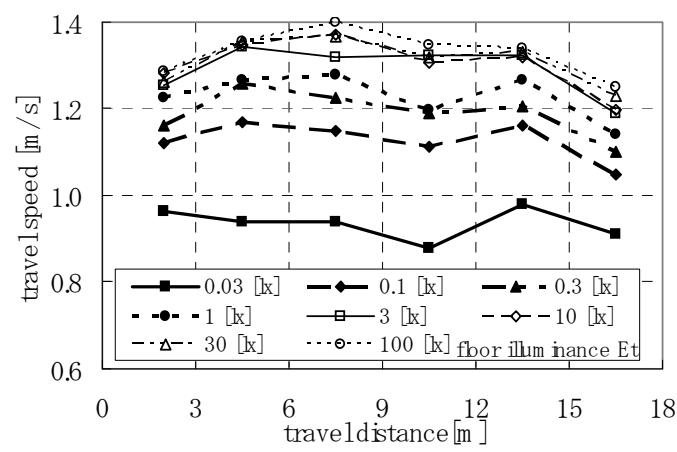

Fig.6 Relationship between travel distance and travel speed (aged group). by age groups is shown in Fig.8. We calculated the visual acuity under each floor illuminance level using Eq.4 and Table 4. In Fig.8, the age difference is not seen, which impliesd that travel speed was does not vary by visual acuity regardless of age. From the relationship shown in Fig.8, the travel speed under 
complete adaptation $\mathrm{v}_{\mathrm{o}}(\mathrm{m} / \mathrm{s})$ could be correlated with visual acuity (VA) as expressed by Eq.5 $\left(\mathrm{R}^{2}=0.80\right.$ and 0.86). If we can predict visual acuity under a disaster situation's light conditions, we can further predict performance, i.e. travel speed of evacuees by this equation.

$v_{o}= \begin{cases}1.56 \times V A^{0.12} & (V A<0.25) \\ 1.32 & (V A \geq 0.25)\end{cases}$

where VA is visual acuity, and $\mathrm{v}_{\mathrm{o}}(\mathrm{m} / \mathrm{s})$ is the travel speed under complete adaptation.

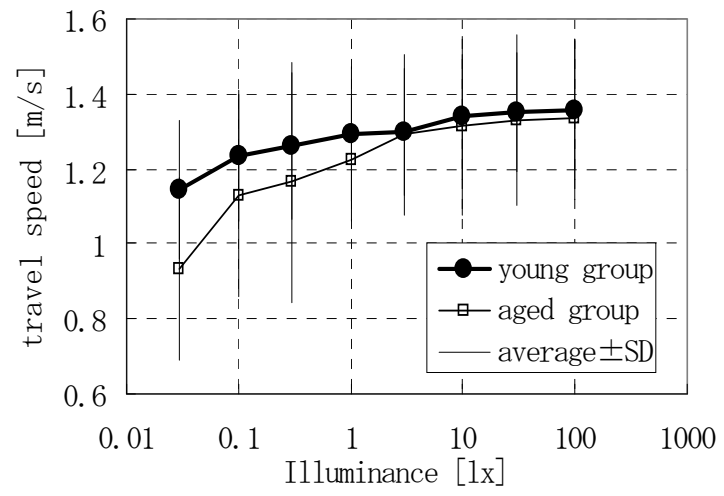

Fig. 7. Relationship between illuminance and travel speed

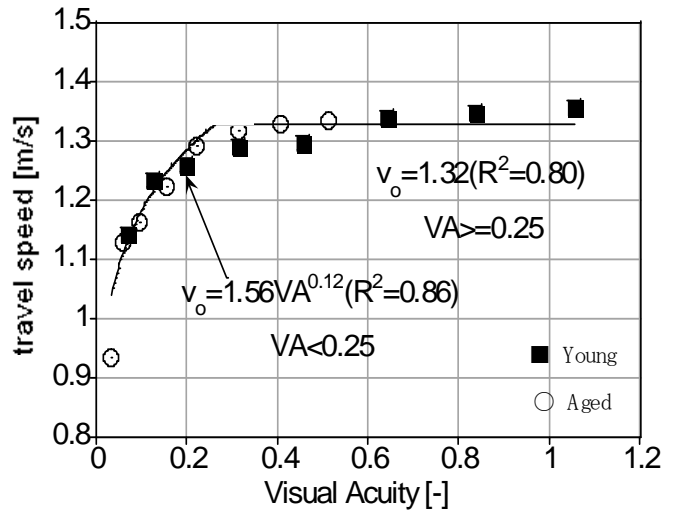

Fig. 8. Relationship between travel speed and visual acuity.

\section{TRAVEL SPEED UNDER INCOMPLETE ADAPTATION}

An example of the relationship between travel distance and speed under incomplete adaptation is shown in Fig.9. When the illuminance in the adaptation space and in the travel space was significantly different, the travel speed increased to the level of complete adaptation's speed as subjects travel farther.

We treated the illuminance difference between the adapting space and the travel space as the ratio of adapting illuminance change, $\mathrm{RE}_{\text {adaptation }}$ expressed in Eq.1. Moreover we defined the ratio of travel speed $\left(R v_{\text {adaptation }}\right)$ regarding the effect of adaptation on travel speed as expressed by Eq.6.

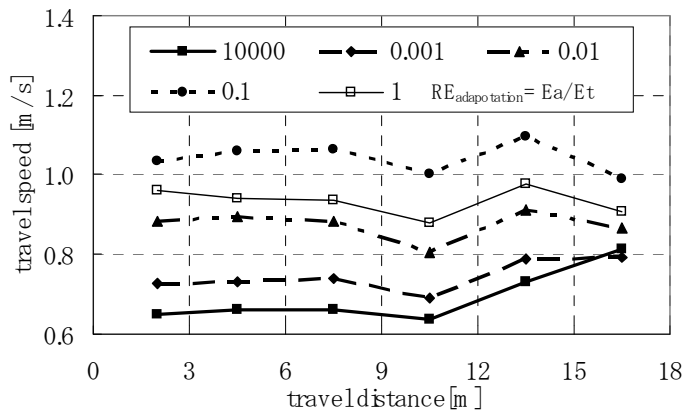

Fig.9 Relationship between travel distance and travel speed under incomplete condition (aged group, $\mathrm{Et}=0.03 \mathrm{~lx}$ ).

$$
R v_{\text {adaptation }}=\frac{v_{i}}{v_{o}}
$$

where $\mathrm{v}_{\mathrm{o}}[\mathrm{m} / \mathrm{s}]$ was the travel speed under complete adaptation, and $\mathrm{v}_{\mathrm{i}}[\mathrm{m} / \mathrm{s}]$ was the travel speed under incomplete adaptation.

The relationship among the illuminance of the travel space $E_{t}$, the ratio of adapting illuminance change $\mathrm{RE}_{\text {adaptation, }}$ and the ratio of travel speed $\mathrm{Rv}_{\text {adaptation }}$ were shown in Fig.10. We calculated the mean value of $R v_{\text {adaptation }}$ based on each subject's result. The value of $R v_{\text {adaptation }}$ should be one or smaller, but the data for individual travel speeds were rather unstable, so some results of $\mathrm{Rv}_{\text {adaptation }}$ were greater than one. On the whole, $E_{t}$ was lower and $\mathrm{RE}_{\text {adaptation }}$ was greater, $\mathrm{RE}_{\text {adaptation }}$ decreased.

The difference seen in Fig.10 could be explained by visual acuity. In Fig.11, the results of $\mathrm{Rv}_{\text {adaptation }}$ under different floor illuminance for both age-groups were plotted versus visual acuity. Under the condition that $\mathrm{RE}_{\text {adaptation }}$ value was 10 (i.e., the illuminance of the adapting space is 10 times that of the travel space), $\mathrm{Rv}_{\text {adaptation }}$ was nearly 1. Regression equations in Fig. 11 were established when $\mathrm{RE}_{\text {adaptation }}$ was more than 
100. The regression relationship between visual acuity and $\mathrm{Rv}_{\text {adaptation }}$ in Fig.11 were given by Eq.7. Combining Eq.7 with Eq.5 could make calculation of travel speed under incomplete adaptation conditions.

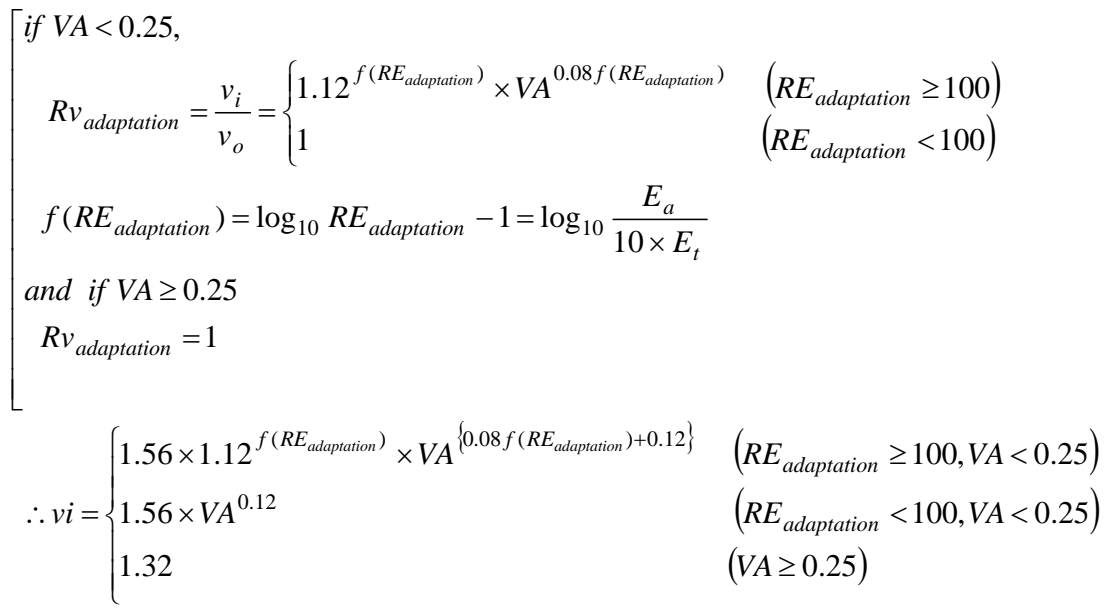

where Ea and Et are the illuminance of the adapting space and the travel space(lx), $\mathrm{v}_{\mathrm{o}}$ and vi are the travel speeds under complete and incomplete adaptations (m/s), and VA is visual acuity, respectively.

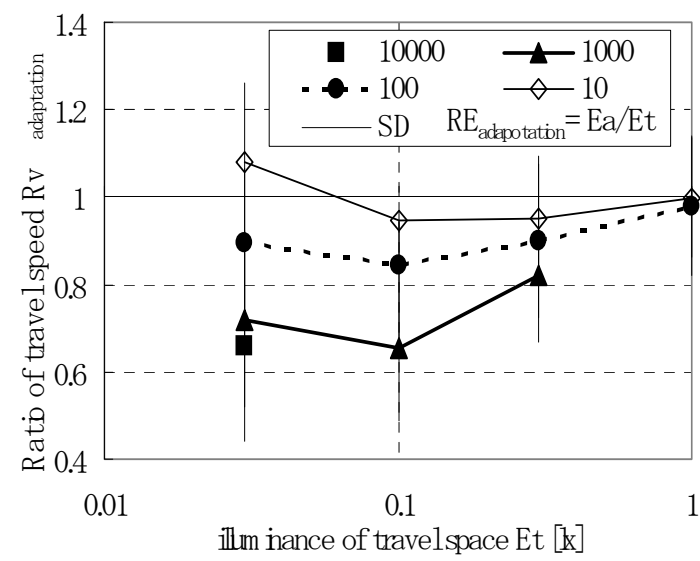

Fig. 10. Relationship between travel speed and $\mathrm{RE}_{\text {adaptation }}$ (aged group).

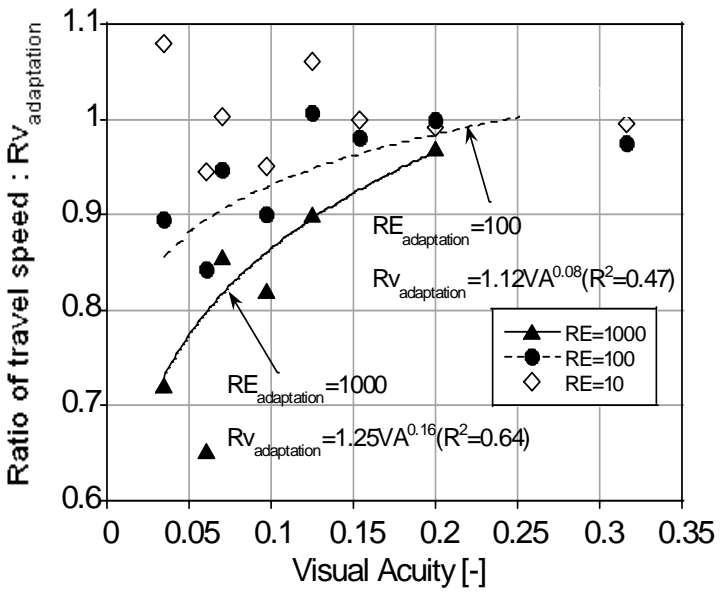

Fig. 11. Relationship between $\mathrm{Rv}_{\text {adaptation }}$ and visual acuity in incomplete adaptation.

\section{TRAVEL SPEED IN SMOKE}

Next, we indicated the result of travel speed under complete adaptation in smoke. Figure 12 shows the comparisons between the results in smoke and the results without smoke. The result showed the large degradation of travel speed in smoke. Under the same illuminance conditions, subjects walked more slowly in smoke than without smoke. In above-mentioned Fig.7, there was little difference between travel speed of the young and aged groups, and both travel speeds were constant under the illuminance condition 3.0[lx] or more. However, under the conditions more than 3.0[lx] in smoke in Fig.12, the travel speeds of both groups increased as illuminance increased. The travel speed of the young group was consistently higher than that of the aged group.

Even under the same illuminance conditions, subjects walked more slowly with smoke than without. Therefore we define the ratio of travel speed in smoke $\left(\mathrm{Rv}_{\mathrm{smoke}}\right)$ regarding the effect of smoke by Eq.8. 


$$
R v_{\text {smoke }}=\frac{v_{s}}{v_{o}}
$$

where $\mathrm{v}_{\mathrm{o}}(\mathrm{m} / \mathrm{s})$ is the travel speed under complete adaptation without smoke, and $\mathrm{v}_{\mathrm{s}}(\mathrm{m} / \mathrm{s})$ is the travel speed under complete adaptation in smoke.

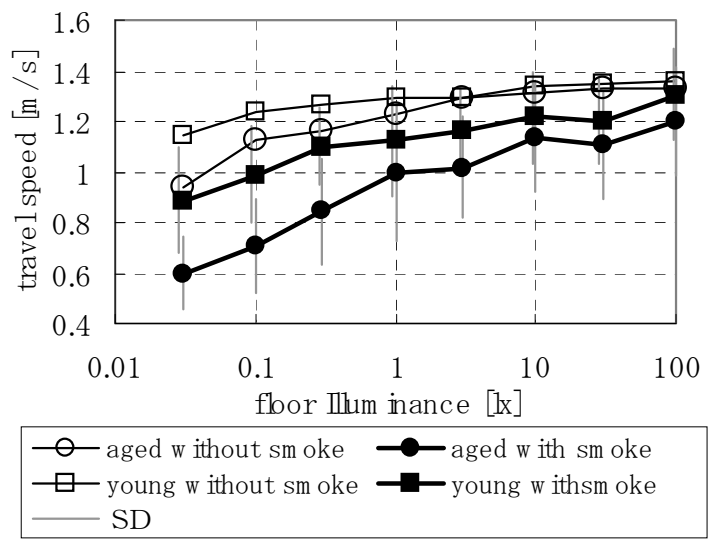

Fig. 12. Comparisons between the results with smoke and the results without smoke.

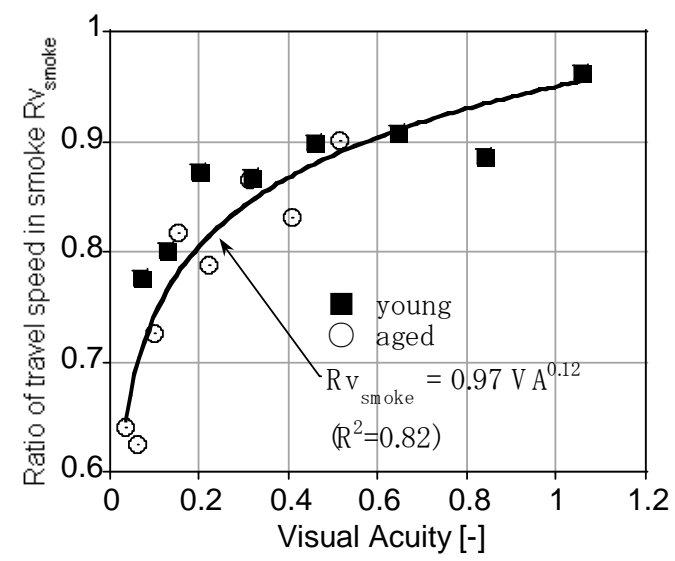

Fig. 13. Relationship between visual acuity and

$\mathrm{Rv}_{\text {smoke }}$ in smoke.

The differences seen in Fig. 12 could be explained by visual acuity, too. The relationship between $\mathrm{Rv}_{\text {smoke }}$ and visual acuity by age groups are shown in Fig.13. We calculated the mean value of $\mathrm{Rv}_{\text {smoke }}$ based on each subject's result, and the visual acuity under each illuminance level using Eq.4 and Table 4. The age difference was not seen in Fig. 13, which implied that the effect of smoke on travel speed could be explained by visual acuity regardless of age.

The relationship between $\mathrm{Rv}_{\text {smoke }}$ and visual acuity were shown in Eq.9 $\left(\mathrm{R}^{2}=0.82\right)$. Eq.5 showed convergence with $\mathrm{VA} \geqq 0.25$, but Eq.9 suggested smoke affects travel speed at higher levels of visibility. Equation 9 was applicable to smoke density $C s=0.68(1 / \mathrm{m})$. Higher density of smoke leads to lower visibility, so $\mathrm{Rv}_{\text {smoke }}$ is predicted to decrease. Also, when smoke density is zero (Cs=0), $\mathrm{v}_{\mathrm{s}}$ equals $\mathrm{v}_{\mathrm{o}}$, so $\mathrm{Rv}_{\text {smoke }}$ is equivalent to 1 . In order to built a parameter of smoke density into Eq.9, we need to add results under different smoke condition.

$$
\begin{aligned}
R v_{\text {smoke }} & =\frac{v_{s}}{v_{o}}=0.97 \times V A^{0.12} \\
\therefore v_{s} & = \begin{cases}1.51 \times V A^{0.24} & (V A<0.25) \\
1.28 \times V A^{0.12} & (V A \geq 0.25)\end{cases}
\end{aligned}
$$

where $\mathrm{v}_{\mathrm{o}}$ and $\mathrm{v}_{\mathrm{s}}$ are the travel speeds under complete adaptations without and with smoke respectively [m/s], and VA is visual acuity.

\section{PSYCHOLOGICAL STATE AND TRAVEL SPEED}

We explored the relationship between travel speed and a subject's psychological state. Figure 14 shows an example of the relationship between floor illuminance and the evaluation ratio of the 30 aged subjects. For all evaluations, the ratio of negative response became smaller, and ratio of "no problem" became greater with higher floor illuminance. In order to focus on conditions creating a negative state, we eliminated "no problem” responses from further consideration. 


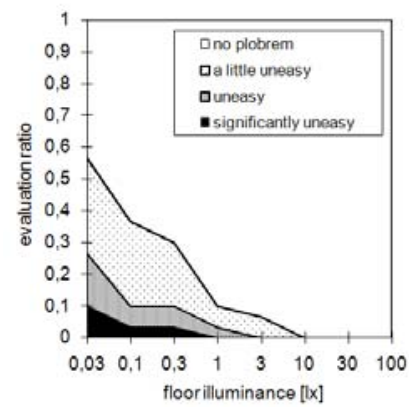

Fig. 14-1 visibility of travel space

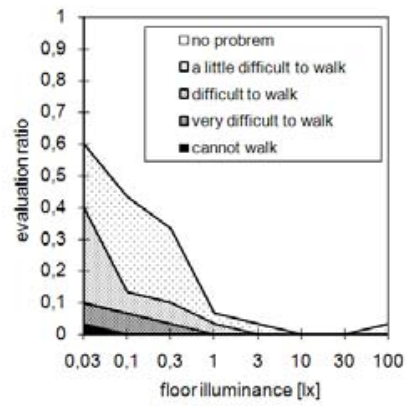

Fig. 14-2 ease to walk or not

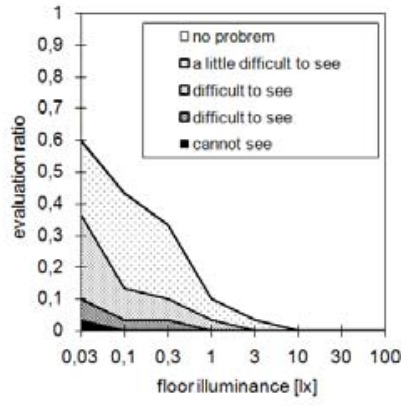

Fig. 14-3 anxiety while walking

Fig.14. Relationship between floor illuminance and evaluation ratio (aged group, without smoke).

Figure 15 shows the relationships between travel speed and the evaluation ratio of negative state by adaptation condition, smoke condition and age group. Without regard to conditions, the general tendency was that as visibility and travel speed increased, negative evaluations decreased. It seemed to show the relationship of travel speed and subjective evaluation in one equation.

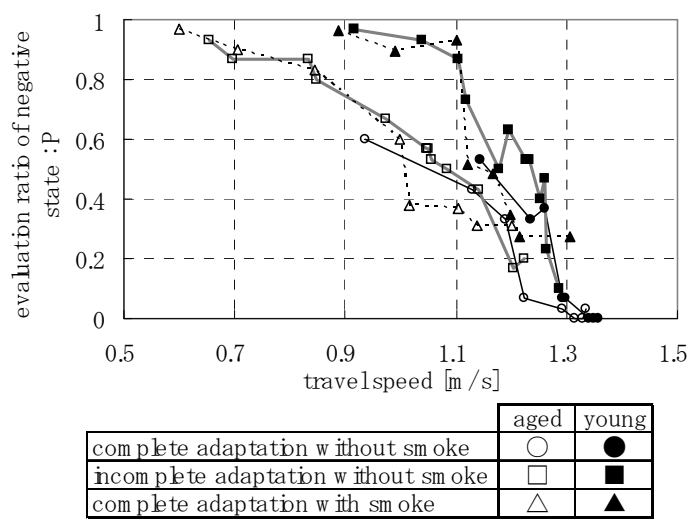

Fig. 15. Relationship between travel speed and negative response ratio of "ease to walking".

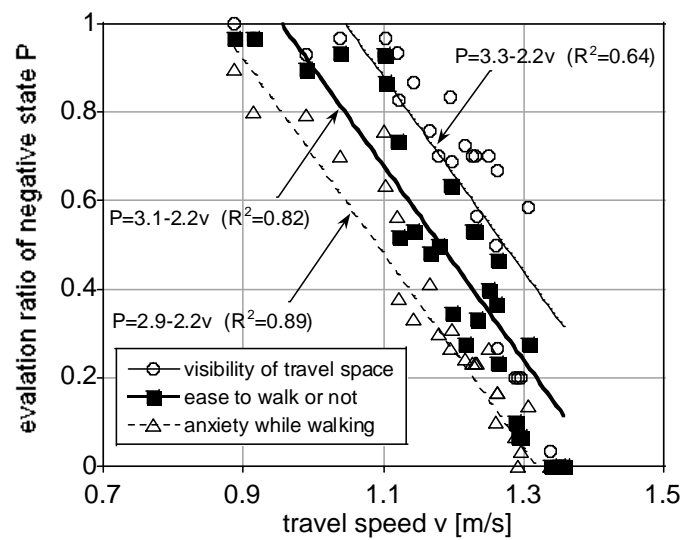

Fig. 16. Relationship between travel speed and negative response ratio for each evaluation (young group).

As aged subjects seemed to avoid negative evaluation in Fig.15, we established Eq.10 indicating a relationship between travel speed and negative response ratio for each evaluation. In each evaluation they showed strong correlation. Negative response was most frequent in evaluations of "visibility of travel space", and least frequent in "anxiety while walking", possibly because anxiety was a result of several factors put together. Under travel speeds less than $1.05(\mathrm{~m} / \mathrm{s})$, all subjects responded negatively in at least one of the evaluations.

$$
P= \begin{cases}k_{1}-2.2 v & \left(v<k_{2}\right) \\ 1 & \left(v \geq k_{2}\right)\end{cases}
$$

where $\mathrm{P}$ is negative response ratio for each evaluation, $\mathrm{v}$ is travel speed [m/s] and $\mathrm{k}_{1}$ and $\mathrm{k}_{2}$ are constant numbers of each psychological state, respectively. In this paper, each value of $k_{2}$ was 1.05 [m/s] (visibility of travel space), $0.95[\mathrm{~m} / \mathrm{s}]$ (easy to walk or not) and $0.86[\mathrm{~m} / \mathrm{s}]$ (anxiety while walking).

\section{CALCULATION MODEL FOR TRAVEL SPEED AND PSYCHOLOGICAL STATE BASED ON VISUAL ACUITY}

Finally, we could construct the calculation model for travel speed and psychological state based on visual acuity shown in Fig.17. The nomenclature in Fig.17 was described in the equations in this paper. It was easy to predict travel speed or psychological state for anyone under various luminous conditions by using Fig.17. In Fig. 17, Fig.E is shown below Fig.C and Fig. D, because it is easy to read the evaluation value 
from any kind of travel speed. In this study, we only set subjects from two age groups. The relationship between visual acuity and age is strong, therefore we can predict the visual acuity of middle age subject using Fig.B.
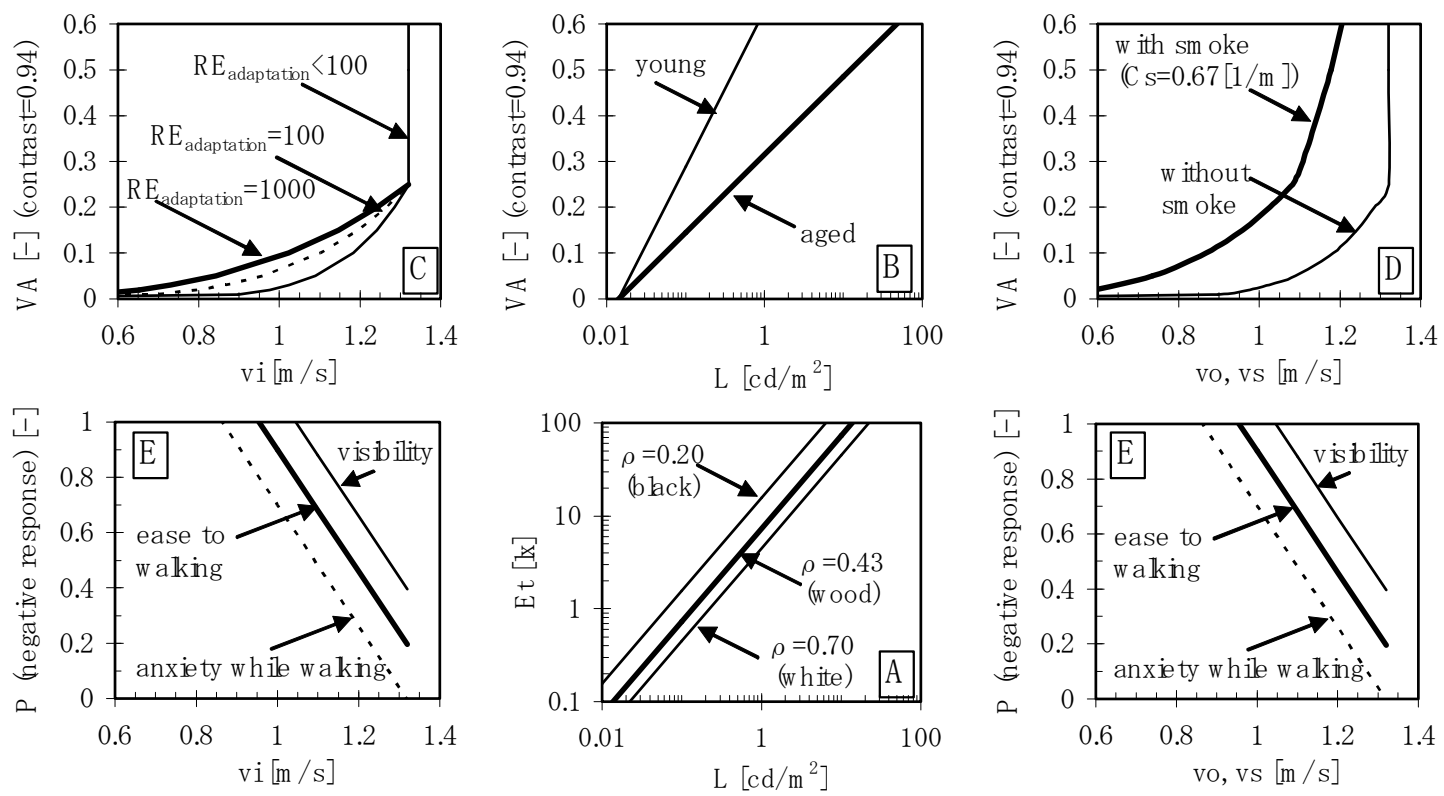

Fig. 17 Diagram of the calculation model for travel speed and psychological state based on visual acuity.

Fig.18 and Fig.19 show two examples of how to use the calculation model for travel speed and psychological state based on visual acuity. Fig.18 shows the procedure for calculating travel speed and psychological state under incomplete adaptation. When the floor illuminance level is $1.0[\mathrm{~lx}]$ in the travel space ( $\rho=0.43$ ), the luminance level may be $0.14\left[\mathrm{~cd} / \mathrm{m}^{2}\right]$ and visual acuity of aged subjects may be 0.17 . If the aged subjects adapted 1000[lx] before entering the travel space, i.e. $\mathrm{RE}_{\text {adaptation }}$ $=1000$, the travel speed $\left(\mathrm{v}_{\mathrm{i}}\right)$ may be $1.19[\mathrm{~m} / \mathrm{s}]$ and $68 \%$ of the aged subjects may negatively evaluate their ability to see. If the aged subjects adapted $30[\mathrm{~lx}]$ before entering the travel space, , i.e. $\mathrm{RE}_{\text {adaptation }}<100$, the travel speed $\left(\mathrm{v}_{\mathrm{i}}\right)$ may be up to $1.26[\mathrm{~m} / \mathrm{s}]$ and $55 \%$ of the aged subjects may negatively evaluate their ability to see. If the subjects are

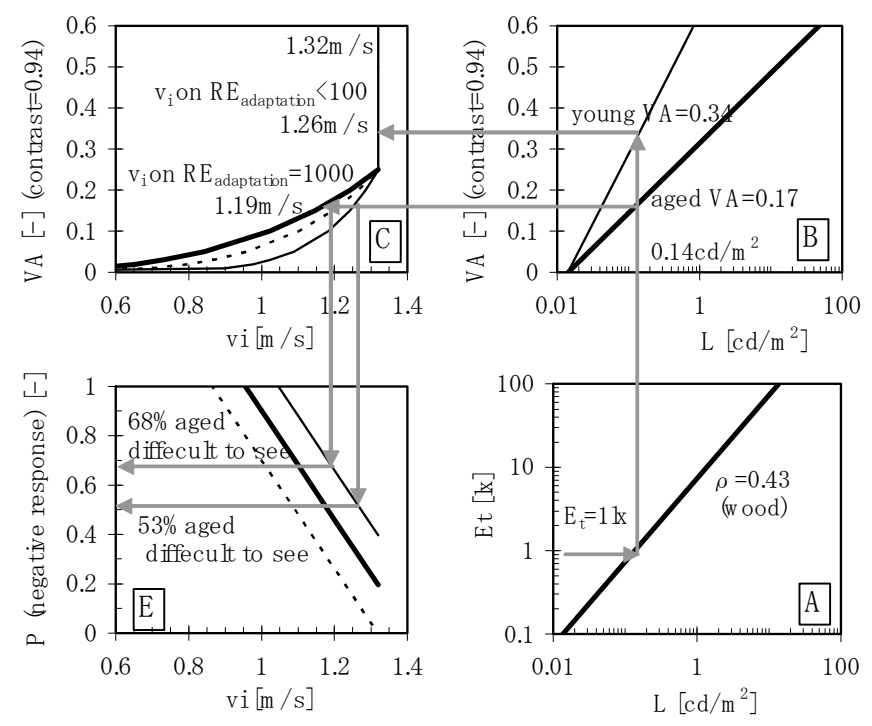

Fig.18. Procedure for calculating travel speed under incomplete adaptation.

young, the travel speed $\left(\mathrm{v}_{\mathrm{s}}\right)$ may be $1.34[\mathrm{~m} / \mathrm{s}]$ regardless of the adaptation. The value of $R E_{\text {adaptation }} \leq 1000$

corresponds actual blackout condition (for example at office room, floor illuminance of normal condition is $1000[\mathrm{~lx}]$ and floor illuminance of blackout turn down to $1[\mathrm{~lx}])$.

Fig.19 showed the procedure for calculating travel speed and psychological state with and without smoke. When the floor illuminance level is $2.0[\mathrm{~lx}]$ in the travel space $(\rho=0.43)$, the luminance level may be $0.27\left[\mathrm{~cd} / \mathrm{m}^{2}\right]$ and visual acuity of young subjects may be 0.44 . Under the situation without smoke, the travel speed $\left(\mathrm{v}_{\mathrm{o}}\right)$ may be $1.32[\mathrm{~m} / \mathrm{s}]$ and no one may have a negative response. Under the situation with smoke, the travel speed $\left(\mathrm{v}_{\mathrm{s}}\right)$ may be down to $1.16[\mathrm{~m} / \mathrm{s}]$ and $35 \%$ subjects of young may have some angst. If subjects 
are aged (over 65 years old), the travel speed $\left(\mathrm{v}_{\mathrm{s}}\right)$ may be down to $1.05[\mathrm{~m} / \mathrm{s}]$ and $60 \%$ of the subjects may have some angst. We can use this diagram for predicting evacuee's performance if the smoke density is less than 0.67 .

\section{CONCLUSIONS}

This paper showed evacuees' performance could be predicted by visual conditions such as the luminous environment and evacuee's visual acuity. Travel speed was determined by visual acuity regardless of age, whether with or without smoke. Under complete adaptation or incomplete adaptation, travel speed decreased if the evacuee's visual acuity was less than 0.25 . Smoke caused the degradation of travel speed even with sufficient illumination. We constructed a calculation model to predict travel speed and psychological

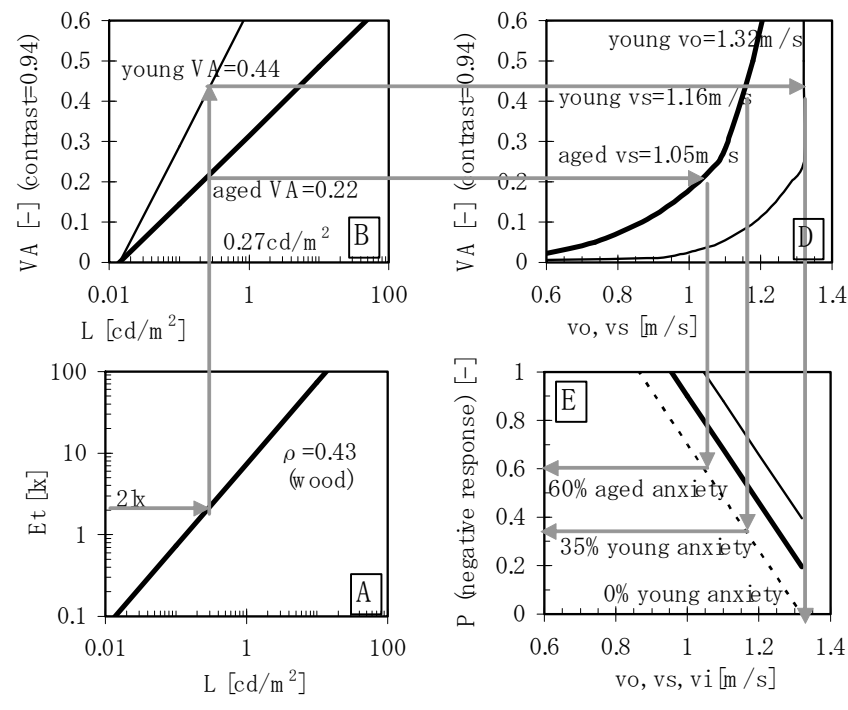

Fig.19. Procedure for calculating travel speed under situation with/without smoke. state based on an evacuee's visual acuity and showed a diagram. If we have to predict visual acuity under the lighting conditions of a fire disaster or blackout, we can further predict performance of evacuees by this model. This experimental passage was easier than a real emergency evacuation. It was straight and walkable, its exit was easy to find, and the subjects walked alone. It will be much more difficult for actual evacuees suffering from fire smoke in a complex urban building. Therefore, this calculation model to predict travel speed consists of many preconditions, so care should be taken when handling.

\section{REFERENCES}

[1] Yuki AKIZUKI and Youko INOUE (2004) The concept of visual acuity ratio to the maximum level of individual visual acuity - The evaluation method of background luminance and visual distance on visibility taking into account of individual visual acuity -, Journal of Light \& Visual Environment, Vol.28, No.1, pp.35-49. doi:10.2150/jlve.28.35

[2] R.C. Simmons (1975) Illuminance, diversity and disability glare in emergency lighting , Lighting Research and Technology, Vol. 7, No.2, pp.121-132. doi:10.1016/0003-6870(76)90040-5

[3] CIE Standards 014-4/E (2007) Emergency lighting

[4] Wolfgang Jaschinski (1982) Conditions of emergency lighting, Ergonomics, Vol.25, pp.363-372. doi:10.1080/00140138208925002

[5] G.M.B.Webber (1984) Emergency lighting recommendations, National Lighting Conference.

[6] Tadahisa Jin (1975) Walking speed in smoke, Bulletin of Japanese Association of Fire Science and Engineering, No.25, Vol.2, pp.44-48 (in Japanese). 